\title{
KMT-2016-BLG-1820 and KMT-2016-BLG-2142: Two Microlensing Binaries Composed of Planetary-mass Companions and Very-low-mass Primaries
}

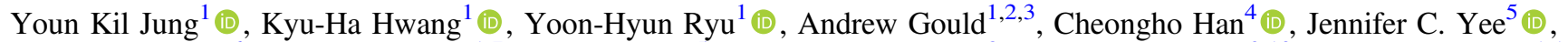

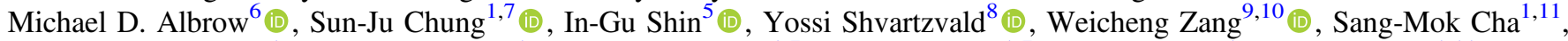 \\ Dong-Jin Kim ${ }^{1}$, Hyoun-Woo Kim ${ }^{1}$ (10, Seung-Lee Kim ${ }^{1,7}$, Chung-Uk Lee ${ }^{1,7}$, Dong-Joo Lee ${ }^{1}$, Yongseok Lee ${ }^{1,11}$, \\ Byeong-Gon Park ${ }^{1,7}$, and Richard W. Pogge ${ }^{2}$ (iD \\ ${ }^{1}$ Korea Astronomy and Space Science Institute, Daejon 34055, Republic of Korea \\ ${ }^{2}$ Department of Astronomy, Ohio State University, 140 W. 18th Ave., Columbus, OH 43210, USA \\ ${ }^{3}$ Max-Planck-Institute for Astronomy, Königstuhl 17, D-69117 Heidelberg, Germany \\ ${ }_{5}^{4}$ Department of Physics, Chungbuk National University, Cheongju 28644, Republic of Korea \\ ${ }^{5}$ Harvard-Smithsonian Center for Astrophysics, 60 Garden St., Cambridge, MA 02138, USA \\ ${ }^{6}$ University of Canterbury, Department of Physics and Astronomy, Private Bag 4800, Christchurch 8020, New Zealand \\ ${ }^{7}$ Korea University of Science and Technology, 217 Gajeong-ro, Yuseong-gu, Daejeon 34113, Republic of Korea \\ ${ }^{8}$ IPAC, Mail Code 100-22, Caltech, 1200 E. California Blvd., Pasadena, CA 91125, USA \\ ${ }^{9}$ Physics Department and Tsinghua Centre for Astrophysics, Tsinghua University, Beijing 100084, People's Republic of China \\ ${ }^{10}$ Department of Physics, Zhejiang University, Hangzhou, 310058, People's Republic of China \\ ${ }^{11}$ School of Space Research, Kyung Hee University, Yongin 17104, Republic of Korea \\ Received 2018 May 24; revised 2018 August 30; accepted 2018 September 18; published 2018 October 18
}

\begin{abstract}
We present the analyses of two short-timescale $\left(t_{\mathrm{E}} \sim 5\right.$ days) microlensing events, KMT-2016-BLG-1820 and KMT-2016-BLG-2142. In both light curves, the brief anomalies were clearly captured and densely covered by the Korea Microlensing Telescope Network survey. From these analyses, we find that both events have small Einstein radii of $\theta_{\mathrm{E}}=0.12$ mas, suggesting that the binary-lens systems are composed of very-low-mass components and/ or are located much closer to the lensed stars than to Earth. From Bayesian analyses, we find that these binaries have total system masses of $0.043_{-0.018}^{+0.043} M_{\odot}$ and $0.088_{-0.041}^{+0.120} M_{\odot}$, implying that they are well within the very-lowmass regime. The estimated lens-component masses indicate that the binary lenses consist of a giant-planet/browndwarf pair (KMT-2016-BLG-1820) and a dark/faint object pair (KMT-2016-BLG-2140) that are located near the deuterium-burning and hydrogen-burning mass limits, respectively. Both lens systems are likely to be in the Galactic disk with estimated distances of about 6 and $7 \mathrm{kpc}$. The projected lens-components separations are 1.1 and $0.8 \mathrm{au}$, and the mass ratios are 0.11 and 0.20 . These prove that the microlensing method is effective to identify these closely separated very-low-mass binaries having low mass-ratios.
\end{abstract}

Key words: binaries: general - brown dwarfs - gravitational lensing: micro

\section{Introduction}

Over the 20 years following the first discoveries by Rebolo et al. (1995) and Nakajima et al. (1995), thousands of very-lowmass (VLM; $M \leqslant 0.2 M_{\odot}$ ) stellar and substellar objects have been discovered. ${ }^{12}$ However, despite the large assemblage of these cool objects, their formation still remains an open question. Various scenarios have been proposed to explain their origins, including ejection from multiple prestellar cores (e.g., Reipurth \& Clarke 2001), turbulent fragmentation of gas in protostellar clouds (e.g., Padoan \& Nordlund 2004), photoionizing radiation from massive nearby stars (e.g., Whitworth \& Zinnecker 2004), and fragmentation of unstable prestellar disks (e.g., Stamatellos \& Whitworth 2009).

Observational studies of VLM binaries can provide effective diagnostics for testing the VLM formation scenarios. This is because the formation mechanisms leave their own traces on the statistical properties of binaries such as frequency, orbit separation, and mass-ratio distributions (e.g., Bate 2009). In addition, binaries can provide a model-independent way to determine physical properties, including masses. Hence, for the comprehensive study of the formation scenarios, it is essential to obtain unbiased VLM binary samples from various detection

\footnotetext{
12 http://www.astro.umontreal.ca/ gagne/listLTYs.php
}

methods that are effective with respect to different population of VLM objects.

Despite its importance, the sample of VLM binaries still remains incomplete. Up until now, the most productive observational method to detect VLM binaries was direct imaging (e.g., Close et al. 2007). Due to its current limit of angular resolution, however, it is difficult to identify close $(\lesssim 3 \mathrm{au})$ binaries by this method, and thus the samples could be biased toward wide binaries (e.g., Burgasser et al. 2007). For the same reason, the method is sensitive to roughly equal mass ratio $\left(q=M_{2} / M_{1} \gtrsim 0.5\right)$ binaries confined to the solar neighborhood and to star-forming regions. Moreover, it is difficult to precisely determine the masses of these objects from spectra because of their faintness and long orbital period. ${ }^{13}$

Microlensing can complement the direct imaging method by detecting binaries that are difficult to identify by other methods. The lensing phenomenon only relies on the gravitational field of a lensing object and thus enables one to detect binaries composed of very faint and even dark objects with masses down to brown dwarf (BD) and planetary regimes. Because

\footnotetext{
${ }^{13}$ We note that VLM binaries discovered from blended-light spectra (e.g., Bardalez Gagliuffi et al. 2014) and from astrometric perturbations (e.g., Sahlmann et al. 2013) have little separation bias. In addition, the astrometric perturbation method is also sensitive to unequal mass ratio binaries.
} 
microlensing events arise on Galactic scales, they can probe binaries located far beyond the solar neighborhood. In addition, the method can detect even tight $(\leqslant 1 \mathrm{au})$ binaries because of its high sensitivity to binaries with small separations. With these advantages, the microlensing technique has discovered various types of VLM objects such as isolated BDs (e.g., Gould et al. 2009; Zhu et al. 2016; Chung et al. 2017), a BD hosting a planetary companion (e.g., Han et al. 2013; Jung et al. 2018), BD-BD binaries (e.g., Choi et al. 2013), and BDs around VLM stars (e.g., Jung et al. 2015; Han et al. 2017).

In this work, we report two binary systems that are composed of planetary-mass companions and VLM primaries. They were discovered from the analysis of short-timescale events found from the Korea Microlensing Telescope Network (KMTNet; Kim et al. 2016) survey conducted in 2016 season.

\section{Observations}

These VLM binaries were discovered in lensing events KMT2016-BLG-1820 and KMT-2016-BLG-2142. The equatorial and galactic coordinates of the events are (R.A., decl.) $)_{\mathrm{J} 2000}=$ $(17: 55: 03.54,-29: 31: 00.91) \quad[(l, b)=(0.56,-2.07)]$ and (R.A ., decl. $)_{\mathrm{J} 2000}=(17: 52: 26.88,-29: 23: 04.42)[(l, b)=(0.38$, $-1.51)$, respectively.

The events were detected from the KMTNet survey. The survey was conducted toward the Galactic bulge field using three identical $1.6 \mathrm{~m}$ telescopes that are globally distributed at the Cerro Tololo Inter-American Observatory in Chile (KMTC), South African Astronomical Observatory in South Africa (KMTS), and Siding Spring Observatory in Australia (KMTA). Both events are located in two overlapping fields (KMTNet BLG02 and BLG42), resulting in a combined $4 \mathrm{hr}^{-1}$ cadence. Most KMTNet images were obtained in $I$-band and some $V$-band images were taken to measure the colors of the lensed stars. For the analysis, the data of the individual events were reduced using the pySIS package (Albrow et al. 2009), and their errors were re-scaled using the method of Yee et al. (2012).

In the summer of 2017, KMT-2016-BLG-1820 and KMT2016-BLG-2142 were initially identified as "clear" and "possible" microlensing events through a human review of lensing candidates found by the KMTNet Event Finder algorithm (Kim et al. 2018a). The light curves used for the algorithm were constructed based on the Difference Imaging Analysis method (DIA; Alard \& Lupton 1998; Woźniak 2000), for which catalog stars are mainly assembled from the OGLEIII star catalog (Szymański et al. 2011). During this inspection, KMT-2016-BLG-1820 and KMT-2016-BLG-2142 were discovered on the light curves of $I=19.4$ and $I=19.8$ catalog stars, respectively. In contrast to KMT-2016-BLG-1820, KMT2016-BLG-2142 was only rated as "possible" (rather than "clear") microlensing at this stage due to its relatively noisy DIA light curve. In the course of carrying out the automated pySIS reductions in preparation for the data release in the winter of 2017 (Kim et al. 2018b), however, it was recognized that the high noise in the DIA light curve originated from a position offset between the true lensed star and the catalog star. From the pySIS light curve, the lensing anomaly of KMT2016-BLG-2142 was then clearly identified.

\section{Analysis}

Figures 1 and 2 show the light curves of KMT-2016-BLG1820 and KMT-2016-BLG-2142, respectively. Both light curves exhibit strong spikes with U-shaped troughs. Also, there exists a weak bump before the spike near $\mathrm{HJD}^{\prime}$ (=HJD - 2450000 days $) \sim 7627$ for KMT-2016-BLG1820 and a strong bump after the spike near $\mathrm{HJD}^{\prime} \sim 7613$ for KMT-2016-BLG-2142. These spikes and bumps are typical binary-lensing anomalies that are generally produced by caustic-crossings and caustic-approaches of lensed stars, respectively. Thus, we investigate the events based on the binary-lens interpretation.

To analyze to the light curve, we use the parameterization and follow the modeling procedure discussed in Jung et al. (2015). We initially conduct a search over a grid of $(s, q, \alpha)$, where $s$ is the projected lens-component separation and $\alpha$ is the trajectory angle. We note that all of the lengths are scaled to the angular Einstein radius of the lens, $\theta_{\mathrm{E}}$. From this initial search, we explore local $\chi^{2}$ minima on the grid-parameter space. Figure 3 shows our derived $\Delta \chi^{2}$ surface over the $(\log s, \log q)$ plane. For KMT-2016-BLG-1820, we find only one local minimum. For KMT-2016-BLG-2142, we identify a pair of local solutions (marked as "Close" and "Wide") for which the mass ratios are similar but the separations have opposite signs of $\log s$, i.e., a close/wide degeneracy (Griest \& Safizadeh 1998; Dominik 1999). To find the global minimum, we then seed these local solutions into new MCMCs and allow all parameters to vary. Note that, in this last stage, we additionally explore the parameter space including the microlens parallax (Gould 1992, 2004) and orbital motion of the lens (Dominik 1998; Jung et al. 2013), but we cannot measure the signals because of the faintness and short timescales of the events.

Table 1 gives the best-fit solutions of the individual events. The corresponding model curves are presented in Figures 1 and 2. Also presented in Figure 4 are the configurations of the lens systems where the source trajectories (straight lines with arrows) with respect to the caustics (red closed curves) and the lens components (two blue circles) are shown. We find that both events have similar lensing characteristics in the sense that the derived Einstein timescales $t_{\mathrm{E}}$ are relatively short $\left(t_{\mathrm{E}} \lesssim 5\right.$ days $)$ and mass ratios are relatively low $(q \lesssim 0.2)$. Considering that $t_{\mathrm{E}}$ is proportional to the lens $\operatorname{mass}\left(\propto \sqrt{M_{\mathrm{tot}}}\right)$, these give a clue that the secondary masses could correspond to those of substellar objects. For each event, we find that the U-shape variation was generated by the source crossing over the resonant caustic that forms when the projected separation is similar to $\theta_{\mathrm{E}}$. The weak bump in KMT-2016-BLG-1820 near $\mathrm{HJD}^{\prime} \sim 7627$ was generated when the source approached one of the cusps located close to the lower-mass lens component, while the strong bump in KMT-2016-BLG-2142 near $\mathrm{HJD}^{\prime} \sim 7613$ was produced when the source approached one of the central cusps located close to the higher-mass component. For KMT-2016-BLG-2142, we find that the "Close" model is favored over the "Wide" model by $\Delta \chi^{2} \sim 41(>6 \sigma)$, which is statistically high enough to exclude the wide-binary interpretation. Hence, we only consider the "Close" solution.

For both events, the finite source effects are clearly detected from which we can measure the normalized source radius $\rho_{*}$. These enable us to determine $\theta_{\mathrm{E}}$ and the lens-source relative 


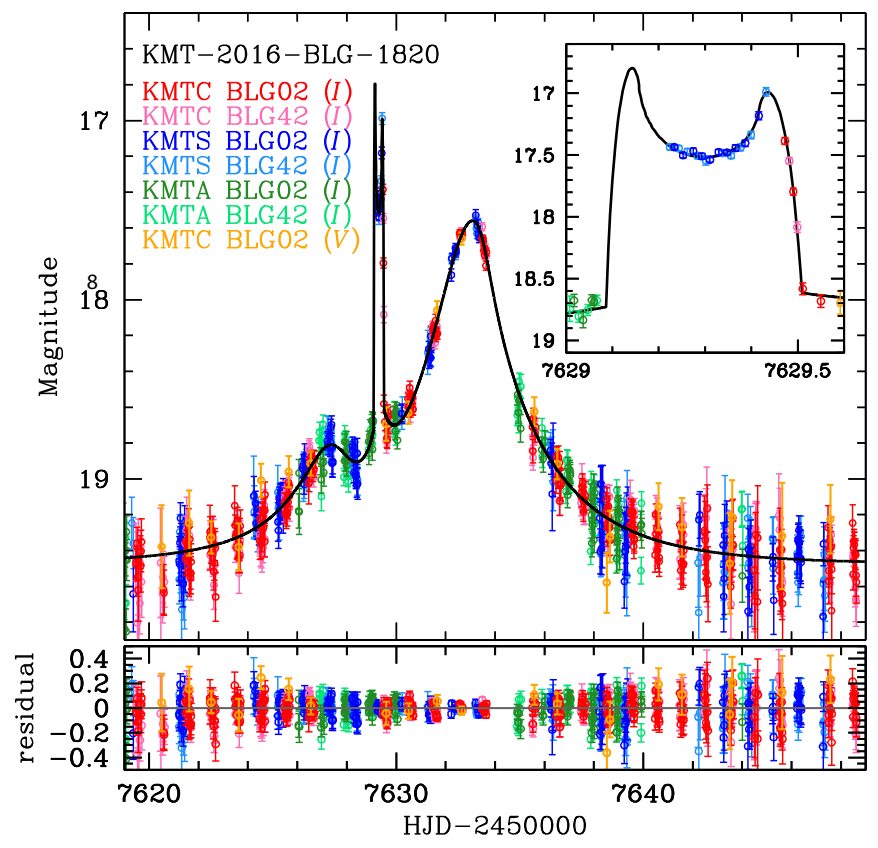

Figure 1. Light curve of KMT-2016-BLG-1820. The right inset shows the caustic crossing centered at $\mathrm{HJD}^{\prime} \sim 7629.3$. The black curve on the data is the best-fit model. The residuals from the model are presented in the lower panel. Note that the $V$-band data are only used for the source color measurement.

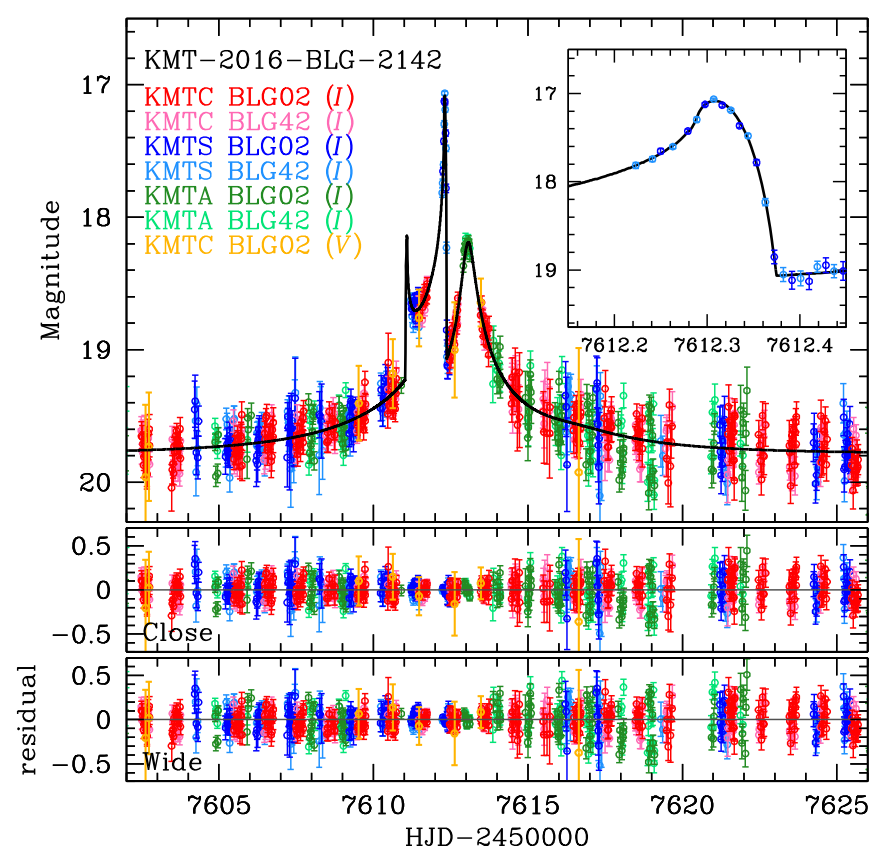

Figure 2. Light curve of KMT-2016-BLG-2142. The right inset shows the caustic exit centered at $\mathrm{HJD}^{\prime} \sim 7612.3$. The black curve on the data is the bestfit "Close" model. The lower two panels show the residuals from the "Close" and "Wide" solutions.

proper motion $\mu$ by

$$
\theta_{\mathrm{E}}=\frac{\theta_{*}}{\rho_{*}} ; \quad \mu=\frac{\theta_{\mathrm{E}}}{t_{\mathrm{E}}},
$$

where $\theta_{*}$ is the angular radius of the source. To determine $\theta_{*}$, we adopt the standard method of Yoo et al. (2004). First, we build the KMTNet star catalog using the pyDIA reductions and calibrate the brightness of stars using the OGLE-III catalog
(Szymański et al. 2011). Second, we estimate the source $(V-I, I)$ from the model and then place the source star on the constructed color-magnitude diagram (CMD). Third, we measure the relative source position using the giant clump (GC) centroid as a reference, i.e., $\Delta(V-I, I)$. Fourth, we estimate the de-reddened source position $(V-I, I)_{0}$ as

$$
(V-I, I)_{0}=\Delta(V-I, I)+(V-I, I)_{0, \mathrm{GC}} \text {. }
$$

Here the de-reddened GC centroid $(V-I, I)_{0, \mathrm{GC}}$ is known from independent observations (Bensby et al. 2013; Nataf et al. 2013). Finally, we deduce $\theta_{*}$ by first converting $(V-I)_{0}$ to $(V-K)_{0}$ from the color-color relation (Bessell \& Brett 1988) and then by applying the $(V-K)_{0}-\theta_{*}$ relation of Kervella et al. (2004). In Table 2, we summarize our derived offsets $\Delta(V-I, I)$, de-reddened GC and source positions, angular source radii, angular Einstein radii, and relative lenssource proper motions of the individual events. In Figure 5, we present the GC and source positions of the individual events in the corresponding CMDs.

The angular Einstein radius is connected to $M_{\text {tot }}$ and the lens distance $D_{\mathrm{L}}$ by

$$
\theta_{\mathrm{E}} \equiv \sqrt{\kappa M_{\mathrm{tot}} \pi_{\mathrm{rel}}} ; \quad \pi_{\mathrm{rel}}=\mathrm{au}\left(\frac{1}{D_{\mathrm{L}}}-\frac{1}{D_{\mathrm{S}}}\right),
$$

where $\kappa=4 G /\left(c^{2} \mathrm{au}\right) \sim 8.14$ mas $/ M_{\odot}, \pi_{\text {rel }}$ is the lens-source relative parallax, and $D_{\mathrm{S}}$ denotes the distance to the source, which is $D_{\mathrm{S}} \sim 8 \mathrm{kpc}$ for a typical bulge star. For a lensing event caused by a low-mass stellar lens located halfway between the observer and a bulge source, the size of $\theta_{\mathrm{E}}$ is then

$$
\theta_{\mathrm{E}} \sim 0.5 \operatorname{mas}\left(\frac{M_{\mathrm{tot}}}{0.3 M_{\odot}}\right)^{1 / 2}\left(\frac{\pi_{\mathrm{rel}}}{0.12 \mathrm{mas}}\right)^{1 / 2}
$$

For our analyzed events, the measured angular Einstein radii, which are in the range $0.11<\theta_{\mathrm{E}} /$ mas $<0.14$, are substantially smaller than the typical value. This suggests that either the mass of the lens is small $\left(M_{\text {tot }} \ll 0.3 M_{\odot}\right)$ or the lens is located at a large distance $\left(D_{\mathrm{LS}} \ll 1 \mathrm{kpc}\right)$, where $D_{\mathrm{LS}}=D_{\mathrm{S}}-D_{\mathrm{L}}$ is the lens-source distance. Among two possibilities, i.e., small $M_{\mathrm{tot}}$ or large $D_{\mathrm{L}}$, the latter would be unlikely because the derived proper motions are in the range of $7.6<\mu /\left(\right.$ mas $\left.\mathrm{yr}^{-1}\right)<10.3$, suggesting that the lenses of both events are likely to be located in the Galactic disk.

\section{Physical Parameters}

In order to directly measure $M_{\text {tot }}$ and $D_{\mathrm{L}}$, it is required to simultaneously detect $\theta_{\mathrm{E}}$ and the microlens parallax $\pi_{\mathrm{E}}$ :

$$
M_{\mathrm{tot}}=\frac{\theta_{\mathrm{E}}}{\kappa \pi_{\mathrm{E}}} ; \quad D_{\mathrm{L}}=\frac{\mathrm{au}}{\pi_{\mathrm{E}} \theta_{\mathrm{E}}+\pi_{\mathrm{S}}},
$$

where $\pi_{\mathrm{S}}=\mathrm{au} / D_{\mathrm{S}}$ is the parallax of the source. For both analyzed events, we measure the Einstein radii, but we cannot measure nor significantly constrain the microlens parallax signals due to the short timescales of the events. Hence, we cannot directly determine the physical lens parameters.

We can nevertheless constrain the physical parameters of the lenses from a Bayesian analysis based on the measured $t_{\mathrm{E}}$ and $\theta_{\mathrm{E}}$. For this, we first generate a large sample of lenses and sources, and distribute them over a model space using the 


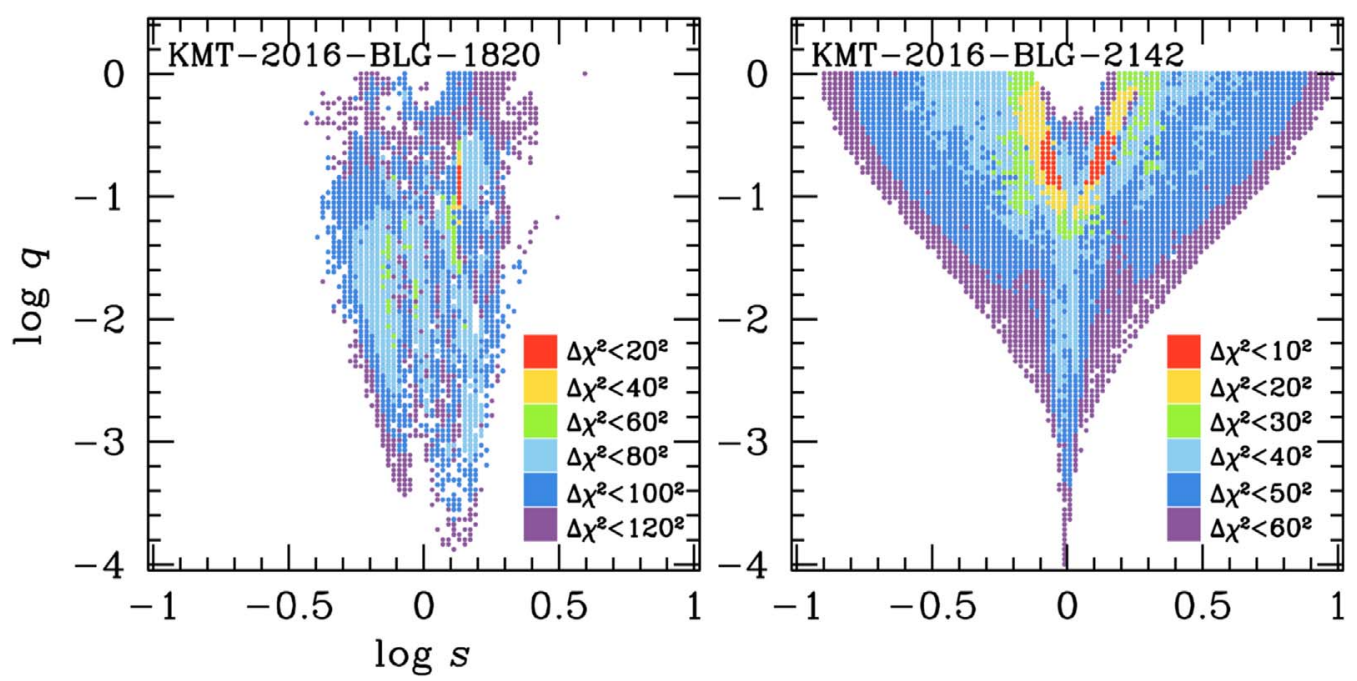

Figure 3. $\Delta \chi^{2}$ distributions in the (log $\left.s, \log q\right)$ space for KMT-2016-BLG-1820 (left panel) and KMT-2016-BLG-2142 (right panel). In both panels, the spaces are divided by $(100,100)$ fixed grids and the inspected ranges are $-1<\log s<1$ and $-4<\log q<0$, respectively. Note that $\Delta \chi^{2}$ contours are differently color coded for each event.

Table 1

Lensing Parameters

\begin{tabular}{lcrr}
\hline \hline \multirow{2}{*}{ Parameters } & \multirow{2}{*}{ KMT-2016-BLG-1820 } & \multicolumn{2}{c}{ KMT-2016-BLG-2142 } \\
\cline { 3 - 4 } & & \multicolumn{1}{c}{ Close } & \multicolumn{1}{c}{ Wide } \\
\hline$\chi^{2} /$ dof & $7510.0 / 7497$ & $6457.1 / 6444$ & $6498.2 / 6444$ \\
$t_{0}\left(\mathrm{HJD}^{\prime}\right)$ & $7632.19 \pm 0.02$ & $7612.54 \pm 0.04$ & $7612.25 \pm 0.06$ \\
$u_{0}$ & $0.24 \pm 0.01$ & $0.14 \pm 0.01$ & $0.15 \pm 0.01$ \\
$t_{\mathrm{E}}($ days $)$ & $4.81 \pm 0.03$ & $5.15 \pm 0.22$ & $6.08 \pm 0.25$ \\
$s$ & $1.40 \pm 0.01$ & $0.97 \pm 0.01$ & $1.21 \pm 0.02$ \\
$q\left(10^{-1}\right)$ & $1.13 \pm 0.03$ & $2.03 \pm 0.11$ & $2.09 \pm 0.11$ \\
$\alpha(\mathrm{rad})$ & $3.04 \pm 0.01$ & $3.96 \pm 0.03$ & $3.94 \pm 0.03$ \\
$\rho_{*}\left(10^{-3}\right)$ & $6.56 \pm 0.21$ & $6.42 \pm 0.34$ & $5.09 \pm 0.21$ \\
\hline
\end{tabular}

Note. $\mathrm{HJD}^{\prime}=\mathrm{HJD}-2450000$ days.

Monte Carlo method. In this process, we adopt the Galactic model of Jung et al. (2018). Next, we investigate the microlensing event rate of each lens-source pair, i.e., $\Gamma \propto \mu \theta_{\mathrm{E}}$. We then explore the posterior distributions of the primary mass $M_{1}$ and $D_{\mathrm{L}}$ by imposing $\Gamma$ and the measured $t_{\mathrm{E}, 1}$ and $\theta_{\mathrm{E}, 1}$ as a prior. ${ }^{14}$ Once $M_{1}$ and $D_{\mathrm{L}}$ are determined, we then estimate the secondary mass $M_{2}$ and the physical primarysecondary projected separation $a_{\perp}$ by

$$
M_{2}=q M_{1}
$$

and

$$
a_{\perp}=s D_{\mathrm{L}} \theta_{\mathrm{E}}
$$

respectively.

In Table 3, we list the physical parameters of the individual events derived from our Bayesian analyses. The corresponding posterior distributions of $M_{1}$ (upper and lower left panels) and $D_{\mathrm{L}}$ (upper and lower right panels) are presented in Figure 6. We note that the physical values and their uncertainties are estimated based on the median values and $68 \%$ confidence intervals of the distributions, respectively.

We find that the total lens masses of the individual events are, respectively, $M_{\mathrm{tot}}=0.043_{-0.018}^{+0.043} M_{\odot}$ and $M_{\mathrm{tot}}=0.088_{-0.041}^{+0.120} M_{\odot}$,

\footnotetext{
${ }^{14} \theta_{\mathrm{E}, 1}=\theta_{\mathrm{E}} / \sqrt{1+q}$ and $t_{\mathrm{E}, 1}=t_{\mathrm{E}} \theta_{\mathrm{E}, 1} / \theta_{\mathrm{E}}$.
}

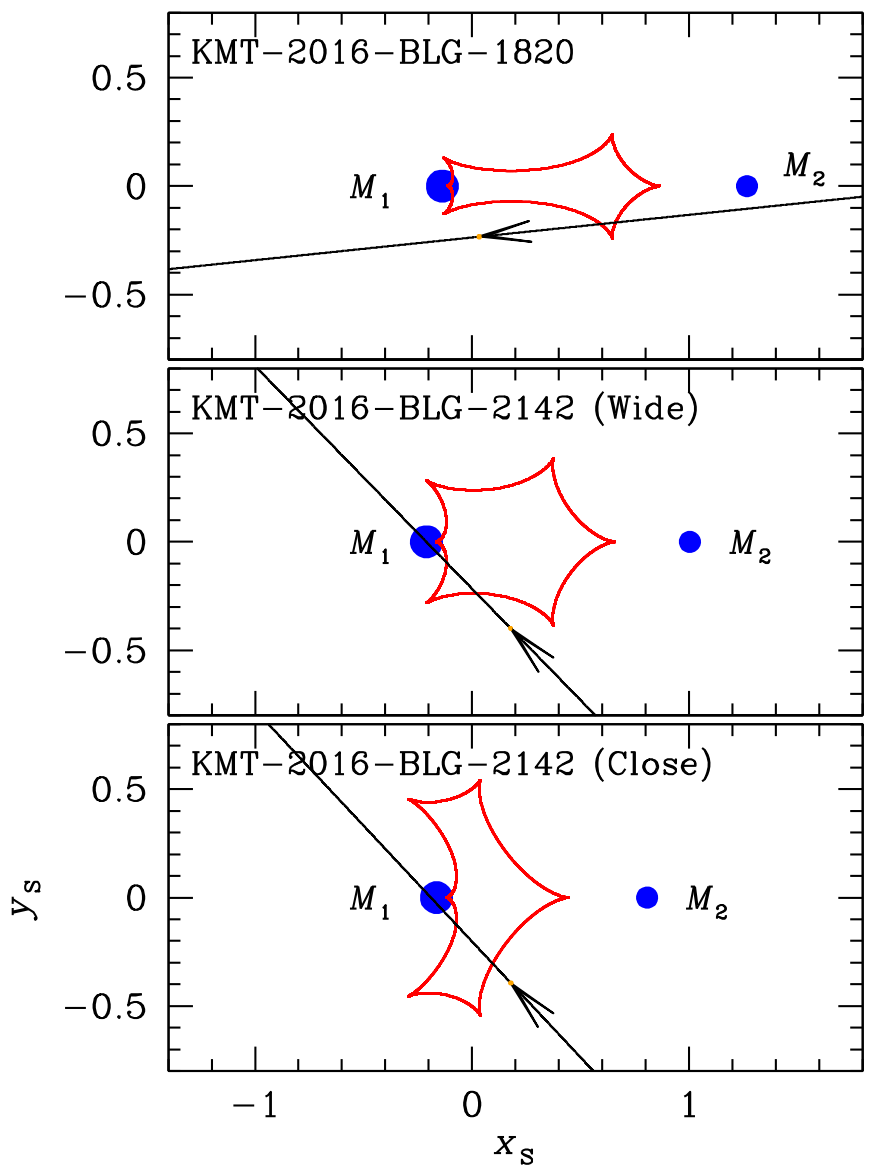

Figure 4. Caustic geometries of KMT-2016-BLG-1820 (upper panel) and KMT-2016-BLG-2142 (middle and lower panels). In each panel, the caustic is represented by the red closed curve. The straight line shows the source trajectory and the arrow on the line indicates the direction of source motion. The two blue circles are the position of primary $\left(M_{1}\right)$ and secondary $\left(M_{2}\right)$ masses.

well within the VLM regime. The binary lens of KMT-2016BLG-1820 is composed of a BD-giant-planet pair. For KMT2016-BLG-2142, the binary host is a faint object whose mass is located near the hydrogen-burning limit $\left(\sim 0.075 M_{\odot}\right.$; Burrows 

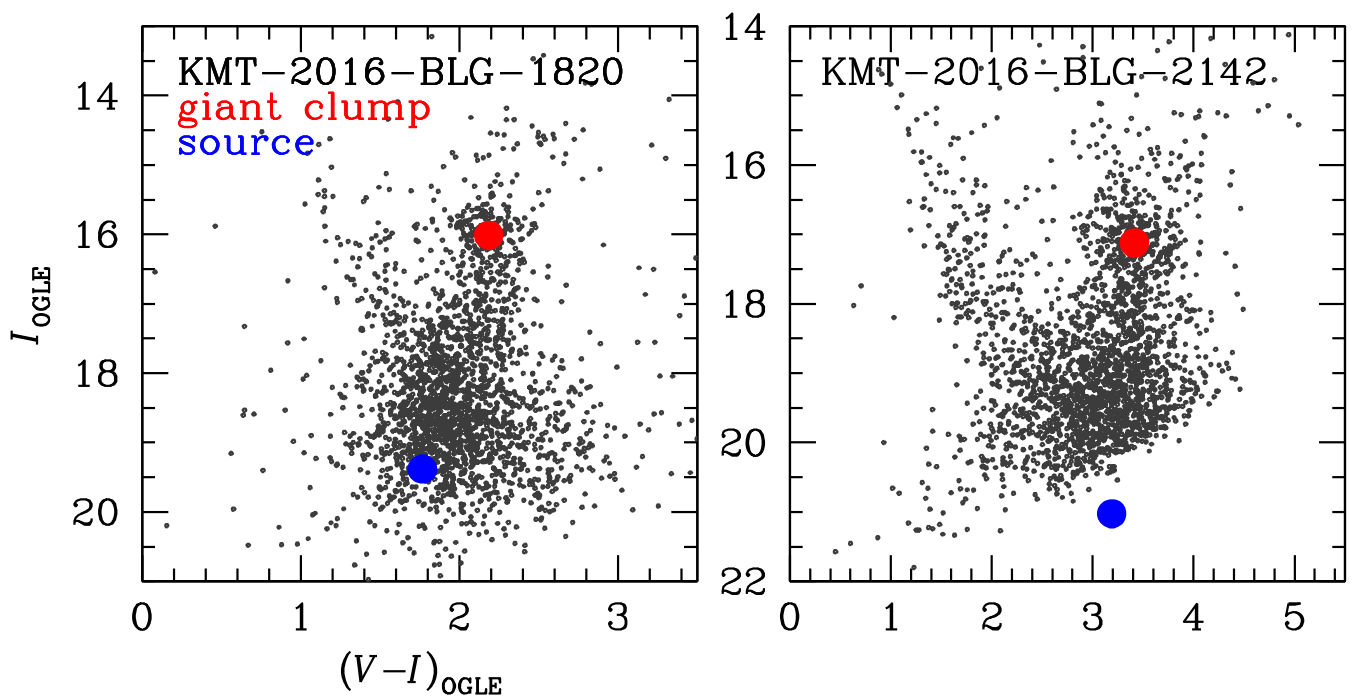

Figure 5. Instrumental color-magnitude diagrams of KMT-2016-BLG-1820 (left panel) and KMT-2016-BLG-2142 (right panel), calibrated to OGLE-III photometry map (Szymański et al. 2011). In each panel, the red and blue dots indicate the positions of giant clump centroid and source, respectively.

Table 2

Source Star Properties

\begin{tabular}{lcr}
\hline \hline Parameters & KMT-2016-BLG-1820 & KMT-2016-BLG-2142 \\
\hline$\Delta(V-I, I)$ & $(-0.42 \pm 0.03,3.37 \pm 0.02)$ & $(-0.23 \pm 0.04,3.90 \pm 0.02)$ \\
$(V-I, I)_{0, \mathrm{GC}}$ & $(1.06 \pm 0.07,14.42 \pm 0.09)$ & $(1.06 \pm 0.07,14.43 \pm 0.09)$ \\
$(V-I, I)_{0}$ & $(0.64 \pm 0.08,17.79 \pm 0.09)$ & $(0.83 \pm 0.08,18.33 \pm 0.09)$ \\
$\theta_{*}(\mu$ as $)$ & $0.807 \pm 0.072$ & $0.781 \pm 0.072$ \\
$\theta_{\mathrm{E}}(\mathrm{mas})$ & $0.123 \pm 0.012$ & $0.122 \pm 0.013$ \\
$\mu$ (mas yr $\left.^{-1}\right)$ & $9.341 \pm 0.882$ & $8.622 \pm 0.934$ \\
\hline
\end{tabular}

Table 3

Physical Parameters

\begin{tabular}{lrr}
\hline \hline Parameters & KMT-2016-BLG-1820 & KMT-2016-BLG-2142 \\
\hline$M_{1}\left(M_{\odot}\right)$ & $0.039_{-0.018}^{+0.043}$ & $0.073_{-0.040}^{+0.117}$ \\
$M_{2}\left(M_{\mathrm{J}}\right)$ & $4.57_{-2.14}^{+5.03}$ & $15.49_{-8.99}^{+24.99}$ \\
$D_{\mathrm{L}}(\mathrm{kpc})$ & $6.26_{-1.28}^{+1.14}$ & $7.01_{-1.16}^{+1.01}$ \\
$a_{\perp}(\mathrm{au})$ & $1.08_{-0.24}^{+0.22}$ & $0.83_{-0.20}^{+0.15}$ \\
\hline
\end{tabular}

et al. 1997), while the companion is a dark object whose mass is located near the deuterium-burning limit $\left(\sim 13 M_{\mathrm{J}}\right.$; Spiegel et al. 2011). The mass ratios and projected separations are $q=0.113 \pm 0.003$ and $a_{\perp}=1.08_{-0.24}^{+0.22}$ au for KMT-2016BLG-1820, and $q=0.203 \pm 0.011$ and $a_{\perp}=0.83_{-0.20}^{+0.15}$ au for KMT-2016-BLG-2142. The lens distances of the individual events are $D_{\mathrm{L}}=6.26_{-1.28}^{+1.14} \mathrm{kpc}$ and $D_{\mathrm{L}}=7.01_{-1.16}^{+1.01} \mathrm{kpc}$, respectively. Both lens systems are likely to be in the Galactic disk, consistent with the prediction based on the relatively high proper motions (see Table 2). Therefore, the small $\theta_{\mathrm{E}}$ values in both events originate from the small lens masses combined with the lens locations being closer to the lensed stars than to Earth.

\section{Discussion}

We found two possible planetary-mass companions around VLM hosts from the analysis of two microlensing events. In both events, the lensing perturbations were clearly captured and densely covered by the KMTNet survey. These prove the capability of KMTNet experiment to identify even brief anomalies in very short-timescale $\left(t_{\mathrm{E}} \sim 5\right.$ day) events.

In Figure 7, we compare the physical properties of these two lens systems to those of previously known VLM $\left(M_{\text {tot }} \leqslant 0.2 M_{\odot}\right)$ binaries and some higher-mass binaries. The figure clearly shows that the fraction of microlensing binaries is high in the low-mass-ratio $(q \leqslant 0.5)$ and close-separation $\left(a_{\perp} \leqslant 1 \mathrm{au}\right)$ regions. Among known VLM binaries discovered from other methods, we find that three binaries have similar mass ratios and total masses to our results: 2MASS J131530942649513 (Burgasser et al. 2011) with $\left(q, M_{\text {tot }} / M_{\odot}\right) \sim(0.27$, 0.073 ), UScoCTIO-108 (Béjar et al. 2008) with $\left(q, M_{\mathrm{tot}} /\right.$ $\left.M_{\odot}\right) \sim(0.20,0.074)$, and 2MASS J1207334-393254 (Chauvin et al. 2004) with $\left(q, M_{\text {tot }} / M_{\odot}\right) \sim(0.20,0.028)$. However, these binaries have wide separations $(>6 \mathrm{au})$ and were discovered in young associations, implying that the sensitivity regimes of microlensing and other detection method are quite different. Hence, these prove that the microlensing method can help to achieve the unbiased VLM binary samples by complementing other methods.

The distinctive sensitivity regimes of microlensing make it possible to improve our understanding of VLM formation mechanisms. From many observational works, it has been suggested that the binary properties of VLM objects are quite different from those of higher-mass stars (e.g., Burgasser et al. 2007). For example, about $15 \% \sim 20 \%$ of VLM systems form as binaries having roughly equal mass ratios with peak separations of $\sim 3 \mathrm{au}$, in contrast to their stellar counterparts for which the binarity frequencies are about $30 \% \sim 60 \%$ and the mass ratios and separations are broadly spread (Duquennoy \& 

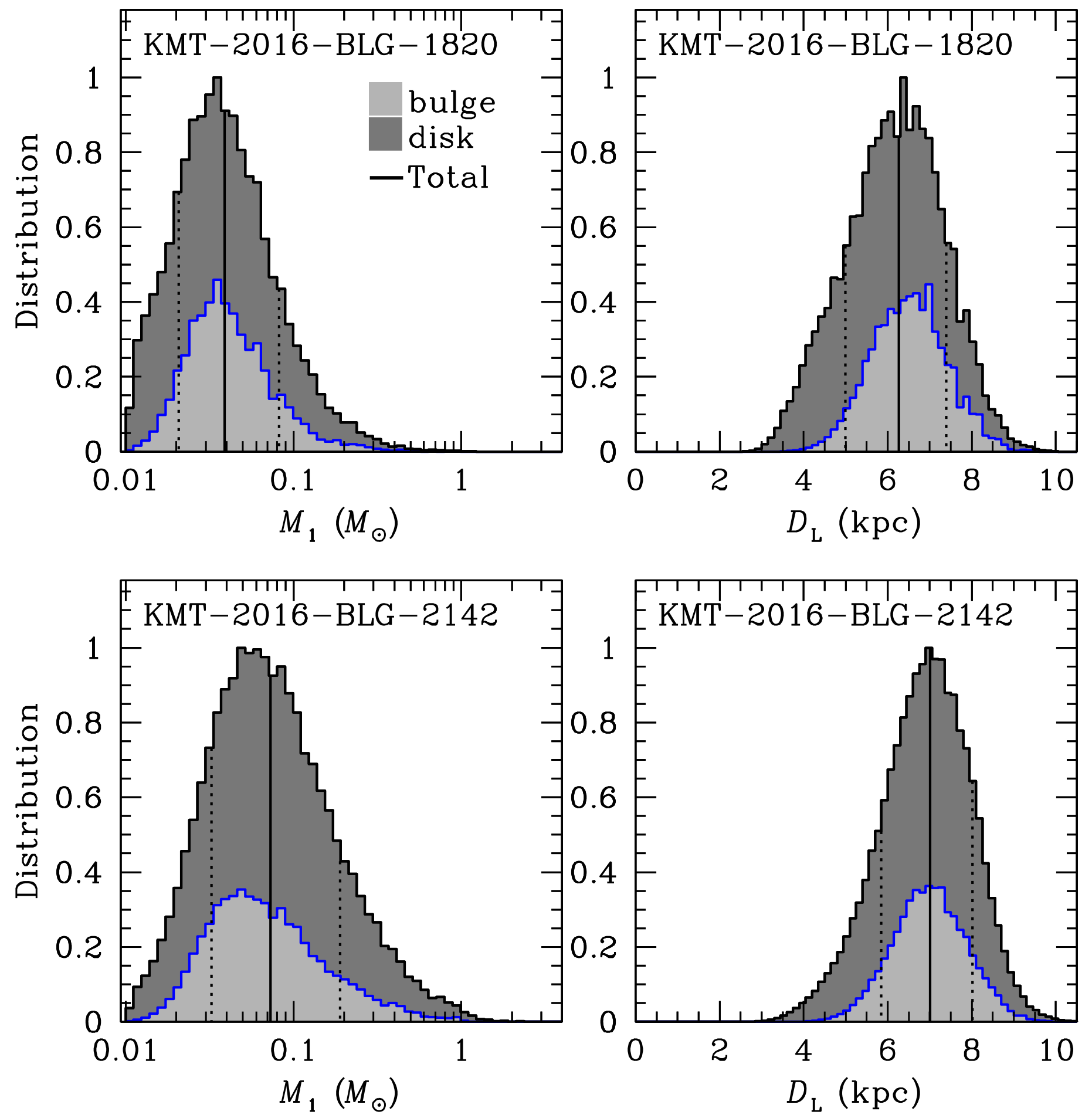

Figure 6. Posterior distributions of $M_{1}$ and $D_{\mathrm{L}}$ for KMT-2016-BLG-1820 (upper left and right panels) and KMT-2016-BLG-2142 (lower left and right panels). In each panel, the total distribution (black line) is divided by bulge (gray) and disk (dark gray) lenses. The vertical solid and dotted lines represent the median value and $68 \%$ confidence intervals, respectively.

Mayor 1991; Fischer \& Marcy 1992). Until now, these statistical differences have been widely used as evidence for distinct formation scenarios between VLM objects and stars. However, the statistical properties of VLM binaries are largely based on the samples collected by direct imaging, implying that the distributions are strongly affected by selection effects and detection biases. In fact, we still do not know clearly whether there exists a significant number of VLM binaries in low-massratio $(q \leqslant 0.5)$ and close-separation $(\leqslant 3 \mathrm{au})$ regions, although it has been suggested that these VLM binaries are as frequent as their counterparts (i.e., high mass ratios and wide separations). Furthermore, understanding the fraction of such VLM binaries is very important, because it provides a key constraint for the question that whether VLM objects form in a similar manner to hydrogen-burning stars or whether they require additional (or different) formation processes. As shown in Figure 7, microlensing can enrich VLM binary samples in these regimes. Hence, the microlensing method can play an 

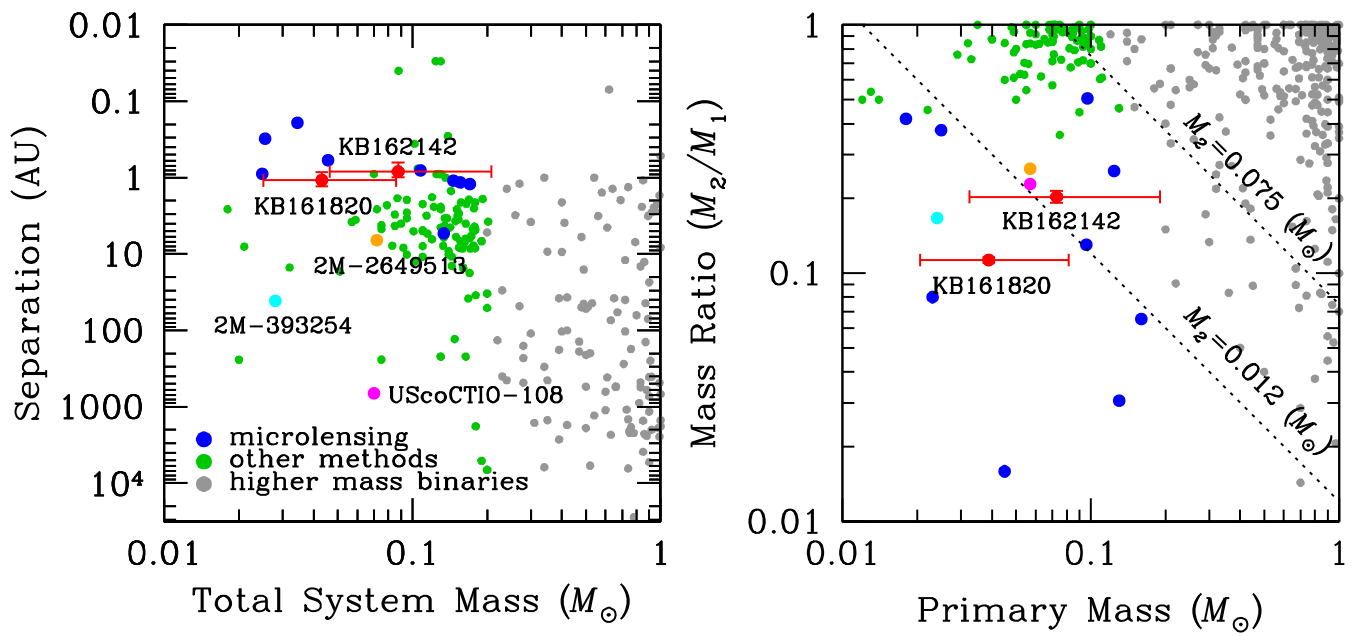

Figure 7. Total mass vs. separation (left panel) and primary mass vs. mass ratio (right panel) distributions for known binaries. The two red dots are the binaries reported in this work. The blue dots are microlensing binaries for which the projected separations are unambiguously determined and the total masses are less than $0.2 M_{\odot}$ (Choi et al. 2013; Han et al. 2013, 2017; Park et al. 2013; Street et al. 2013; Jung et al. 2015, 2018). The green dots are VLM binaries discovered by other methods, while the gray dots are some higher-mass binaries. The yellow, magenta, and cyan dots are the three binaries that have similar mass ratios and total masses to the two reported binaries. The values are obtained from the Very-Low-Mass Binaries Archive (http://www.vlmbinaries.org) and other references (Basri \& Martín 1999; Lane et al. 2001; Burgasser et al. 2008, 2011, 2012, 2016; Allers et al. 2009, 2010; Dhital et al. 2011; Faherty et al. 2011; Liu et al. 2011, 2012; Duchêne et al. 2013; Luhman 2013; Sahlmann et al. 2013; Dupuy et al. 2016; Dupuy \& Liu 2017). For given primary masses $\left(M_{1}\right)$ and mass ratios $(q)$, companion masses $\left(M_{2}\right)$ corresponding to star/brown-dwarf and brown-dwarf/planet boundaries are presented by two dotted lines.

important role in providing the empirical constraints for exploring the origins of VLM objects.

This research has made use of the KMTNet system operated by the Korea Astronomy and Space Science Institute (KASI) and the data were obtained at three host sites of CTIO in Chile, SAAO in South Africa, and SSO in Australia. C.H. was supported by grant 2017R1A4A1015178 of the National Research Foundation of Korea. Work by W.Z. and A.G. were supported by AST-1516842 from the US NSF. W.Z. and A.G. were supported by JPL grant 1500811 . A.G. is supported from KASI grant 2016-1-832-01.

\section{ORCID iDs}

Youn Kil Jung (D) https://orcid.org/0000-0002-0314-6000 Kyu-Ha Hwang (iD https://orcid.org/0000-0002-9241-4117 Yoon-Hyun Ryu (iD https://orcid.org/0000-0001-9823-2907 Cheongho Han (D) https://orcid.org/0000-0002-2641-9964 Jennifer C. Yee (iD https://orcid.org/0000-0001-9481-7123 Michael D. Albrow (i) https://orcid.org/0000-0003-3316-4012 Sun-Ju Chung (Di) https://orcid.org/0000-0001-6285-4528 In-Gu Shin (1) https://orcid.org/0000-0002-4355-9838 Yossi Shvartzvald (1) https://orcid.org/0000-0003-1525-5041 Weicheng Zang (i) https://orcid.org/0000-0001-6000-3463 Hyoun-Woo Kim (iD https://orcid.org/0000-0001-8263-1006 Richard W. Pogge (i) https://orcid.org/0000-0003-1435-3053

\section{References}

Alard, C., \& Lupton, R. H. 1998, ApJ, 503, 325

Albrow, M. D., Horne, K., Bramich, D. M., et al. 2009, MNRAS, 397, 2099 Allers, K. N., Liu, M. C., Dupuy, T. J., \& Cushing, M. C. 2010, ApJ, 715, 561 Allers, K. N., Liu, M. C., Shkolnik, E., et al. 2009, ApJ, 697, 824

Bardalez Gagliuffi, D. C., Burgasser, A. J., Gelino, C. R., et al. 2014, ApJ, 794, 143

Basri, G., \& Martín, E. L. 1999, AJ, 118, 2460

Bate, M. R. 2009, MNRAS, 397, 232

Béjar, V. J. S., Zapatero Osorio, M. R., Pérez Garrido, A., et al. 2008, ApJ, 673,185
Bensby, T., Yee, J. C., Feltzing, S., et al. 2013, A\&A, 549, 147 Bessell, M. S., \& Brett, J. M. 1988, PASP, 100, 1134

Burgasser, A. J., Blake, C. H., Gelino, C. R., et al. 2016, ApJ, 827, 25 Burgasser, A. J., Liu, M. C., Ireland, M. J., et al. 2008, ApJ, 681, 579 Burgasser, A. J., Luk, C., Dhital, S., et al. 2012, ApJ, 757, 110

Burgasser, A. J., Reid, I. N., Siegler, N., et al. 2007, in Protostars and Planets V, ed. B. Reipurth, D. Jewitt, \& K. Keil (Tucson, AZ: Univ. Arizona Press), 427

Burgasser, A. J., Sitarski, B. N., Gelino, C. R., et al. 2011, ApJ, 739, 49 Burrows, A., Marley, M., Hubbard, W. B., et al. 1997, ApJ, 491, 856 Chauvin, G., Lagrange, A.-M., Dumas, C., et al. 2004, A\&A, 425, L29 Choi, J.-Y., Han, C., Udalski, A., et al. 2013, ApJ, 768, 129

Chung, S.-J., Zhu, W., Udalski, A., et al. 2017, ApJ, 838, 154

Close, L. M., Zuckerman, B., Song, I., et al. 2007, ApJ, 660, 1492

Dhital, S., Burgasser, A. J., Looper, D. L., \& Stassun, K. G. 2011, AJ, 141, 7

Dominik, M. 1998, A\&A, 329, 361

Dominik, M. 1999, A\&A, 349, 108

Duchêne, G., Bouvier, J., Moraux, E., et al. 2013, A\&A, 555, A137

Dupuy, T. J., Forbrich, J., Rizzuto, A., et al. 2016, ApJ, 827, 23

Dupuy, T. J., \& Liu, M. C. 2017, ApJS, 231, 15

Duquennoy, A., \& Mayor, M. 1991, A\&A, 248, 485

Faherty, J. K., Burgasser, A. J., Bochanski, J. J., et al. 2011, A\&A, 141, 71

Fischer, D. A., \& Marcy, G. W. 1992, ApJ, 396, 178

Gould, A. 1992, ApJ, 392, 442

Gould, A. 2004, ApJ, 606, 319

Gould, A., Udalski, A., Monard, B., et al. 2009, ApJL, 698, L147

Griest, K., \& Safizadeh, N. 1998, ApJ, 500, 37

Han, C., Jung, Y. K., Udalski, A., et al. 2013, ApJ, 778, 38

Han, C., Udalski, A., Sumi, T., et al. 2017, ApJ, 843, 59

Jung, Y. K., Han, C., Gould, A., \& Maoz, D. 2013, ApJL, 768, L7J

Jung, Y. K., Udalski, A., Gould, A., et al. 2018, AJ, 155, 219

Jung, Y. K., Udalski, A., Sumi, T., et al. 2015, ApJ, 798, 123

Kervella, P., Thévenin, F., Di Folco, E., \& Ségransan, D. 2004, A\&A, 426, 297

Kim, D.-J., Kim, H.-W., Hwang, K.-H., et al. 2018a, AJ, 155, 76

Kim, H.-W., Hwang, K.-H., Kim, D.-J., et al. 2018b, AJ, submitted (arXiv:1804.03352)

Kim, S.-L., Lee, C.-U., Park, B.-G., et al. 2016, JKAS, 49, 37

Lane, B. F., Zapatero Osorio, M. R., Britton, M. C., et al. 2001, ApJ, 560, 390

Liu, M. C., Delorme, P., Dupuy, T. J., et al. 2011, ApJ, 740, 108

Liu, M. C., Dupuy, T. J., Bowler, B. P., et al. 2012, ApJ, 758, 57 Luhman, K. L. 2013, ApJL, 767, L1

Nakajima, T., Oppenheimer, B. R., Kulkarni, S. R., et al. 1995, Natur, 378, 463

Nataf, D. M., Gould, A., Fouqué, P., et al. 2013, ApJ, 769, 88

Padoan, P., \& Nordlund, A. 2004, ApJ, 617, 559

Park, H., Udalski, A., Han, C., et al. 2013, ApJ, 778, 134 
Rebolo, R., Zapatero Osorio, M. R., \& Martín, E. L. 1995, Natur, 377, 129 Reipurth, B., \& Clarke, C. 2001, AJ, 122, 432

Sahlmann, J., Lazorenko, P. F., Ségransan, D., et al. 2013, A\&A, 556, 133

Spiegel, D. S., Burrows, A., \& Milsom, J. A. 2011, ApJ, 727, 57

Stamatellos, D., \& Whitworth, A. P. 2009, MNRAS, 392, 413

Street, R., Choi, J.-Y., Tsapras, Y., et al. 2013, ApJ, 763, 67
Szymański, M. K., Udalski, A., Soszyński, I., et al. 2011, AcA, 61, 83 Whitworth, A. P., \& Zinnecker, H. 2004, A\&A, 427, 299

Woźniak, P. R. 2000, AcA, 50, 42

Yee, J. C., Shvartzvald, Y., Gal-Yam, A., et al. 2012, ApJ, 755, 102

Yoo, J., DePoy, D. L., Gal-Yam, A., et al. 2004, ApJ, 603, 139

Zhu, W., Calchi Novati, S., Gould, A., et al. 2016, ApJ, 825, 60 